\title{
Pattern of Injury with a Graded Excitotoxic Insult and Ensuing Chronic Medial Septal Damage in the Rat Brain
}

\author{
Manuel J. Rodríguez, Alberto Prats,, Yolanda Malpesa, Noemí Andrés,' Marco Pugliese, \\ Montserrat Batlle, and Nicole Mahy ${ }^{1}$
}

\begin{abstract}
Brain damage caused by an acute injury depends on the initial severity of the injury and the time elapsed after the injury. To determine whether these two variables activate common mechanisms, we compared the response of the rat medial septum to insult with a graded series of concentrations of $\alpha$-amino-3-hydroxy-5-methyl-4isoxazole propionic acid (AMPA) with the time-course effects of a low dose of AMPA. For this purpose we conducted a dose-response study at concentrations of AMPA between 0.27 and $10.8 \mathrm{nmol}$ to measure atrophy of the septal area, losses of cholinergic and GABAergic neurons, astroglial and microglial reactions, and calcification. Cholinergic neurons, whose loss paralleled the degree of septal atrophy produced by AMPA, are more sensitive than GABAergic neurons to the injury produced by AMPA. At doses of AMPA above $2.7 \mathrm{nmol}$, calcification and the degree of microglial reaction increased only in the GABAergic region of the septal area, whereas atrophy and neuronal loss reached a plateau. We chose the 2.7-nmol dose of AMPA to determine how these parameters were modified between 4 days and 6 months after injection. We found that atrophy and neuronal loss increased progressively through the 6-month study period, whereas astrogliosis ceased to be observed after 1 month, and calcium precipitates were never detected. We conclude that septal damage does not increase with the intensity of an excitotoxic insult. Rather, it progresses continuously after the insult. Because these two situations involve different mechanisms, short-term paradigms are inappropriate for interpreting the pathogenic mechanisms responsible for long-term neurodegenerative processes.
\end{abstract}

Key words: adult brain injury; immunohistochemistry; in-vivo studies; neurodegenerative disorders; neuronal cell death

\section{Introduction}

A CUTE BRAIN INJURY IS CHARACTERIZED by immediate, massive neuronal death and marked tissue injury occurring through interdependent mechanisms such as glutamate-mediated excitotoxicity, failure of cellular energetics, acidosis, the generation of reactive oxygen species, and a potent inflammatory response (Hazell, 2007; Baptiste and Fehlings, 2006; van Landeghem et al., 2006; Arundine and Tymianski, 2004; Sapolsky, 2001). Acute brain injury can also activate a neurodegenerative process whose effects are timedependent and related to the initial severity of insult to the central nervous system (CNS). Thus, for example, acute neurological injury and chronic brain damage have been linked in boxers, with a correlation between the prevalence of subdural hematoma and dementia pugilistica (Miele et al., 2006; Yoshiyama et al., 2005). The same occurs with the disruption of the blood-brain barrier induced by a focus of epileptic activity, which triggers delayed neurodegeneration and functional cerebral impairment (Tomkins et al., 2007). Cerebrovascular diseases and processes initiated by ischemiareperfusion may also be central in the pathogenesis of Alzheimer's disease (Zlokovic, 2005; Koistinaho and Koistinaho, 2005; Pluta, 2004). Neurodegenerative disorders are characterized by distinct clinical features that result from a discrete and ongoing neuronal loss, leading over periods of years to specific structural alterations in the brain and various forms of cerebral dysfunction (Zlokovic, 2005; Rao and Weiss, 2004). To investigate the complex relationship between a graded acute brain insult and the pathogenic mechanisms activated in chronic neurodegeneration in humans, it is necessary to compare anatomical and cellular parameters in an animal model related to a human disorder (Carvey et al., 2006; Stefanova et al., 2005). Because they can exacerbate cerebral

${ }^{1}$ Unitat de Bioquímica, and ${ }^{2}$ Departament d'Obstetrícia, Ginecologia, Pedriatria, Radiologia i Medicina Funcional, Institut d'Investigacions Biomèdiques August Pi i Sunyer (IDIBAPS), Facultat de Medicina. Universitat de Barcelona, Barcelona, Spain. 
damage, including that caused by oxidative stress and excitatory amino acids (McEwen, 1992; Stein-Behrens et al., 1992), glucocorticoids (GCs) may participate in the common brain response to acute and chronic cerebral lesions. As an example of this is that increased secretion of corticosterone, the primary GC in rodents, decreases hippocampal volume, the length of apical dendrites, and the number of synapses in the CA3 area of the hippocampus (Tata et al., 2006; Sousa et al., 2000). However, this cannot be considered a universal response because of unexpected proinflammatory effects evident in several brain regions (Sorrells and Sapolsky, 2007, for a review).

Degeneration of neurons in the basal forebrain is responsible for neurological deficits in both attention and specific aspects of memory (Etienne et al., 1986; Arendt et al., 1985). To simulate these impairments, animal modeling has focused on acute in-vivo lesions of brain areas related to the hippocampus in different experimental approaches to activate a neurodegenerative process (Ramonet et al., 2004a; Ginsberg et al., 1999; Leutgeb and Mizumori, 1999). Thus, the acute microinjection of excitatory amino acids into the rat medial septum and the diagonal band of Broca (DBB) induces neurodegenerative effects related to the hippocampus. The behavioral deficits associated with cognitive processing (Leutgeb and Mizumori, 1999; Brandner, 1998) reflect a hippocampal reduction in cholinergic activity (Zapata et al., 2000), and of GABAergic and glutamatergic transmission (Rodríguez et al., 2005; Waite et al., 1994).

The aim of the present study was to characterize the anatomical and cellular effects of increasing acute excitotoxic insults to the medial septum, and to determine whether the ongoing process induced by these insults produces similar modifications over a prolonged period. To this end we undertook a dose-response study of $\alpha$-amino-3-hydroxy-5methyl-4-isoxazole propionic acid (AMPA) at concentrations between 0.27 and $10.8 \mathrm{nmol}$, and a time-course study between 4 days and 6 months after AMPA-induced lesioning to determine atrophy of the septal area, neuronal loss, astroglial and microglial reactions, and brain calcification on a quantitative basis. We evaluated the putative relationship between these parameters through the use of correlation analysis and three-dimensional (3-D) reconstruction. We also investigated the participation of GC receptors in the neurodegenerative process.

\section{Methods}

\section{Animals and materials}

These experiments were performed with 2-month-old male Sprague-Dawley rats (Charles River Laboratories, Barcelona, Spain) weighing 250-300 g at the beginning of the study. The animals were kept on a 12-hour light/12-hour dark cycle and housed with free access to food and water. All animals were treated according to the standards of the European Economic Community (86/609/EEC) for animal handling and experimentation. The procedures used in the study were approved by the Ethics Committee of the Universitat de Barcelona, under the supervision of the Generalitat de Catalunya; all efforts were made to minimize animal suffering and to use only the number of animals necessary to yield reliable scientific data. AMPA, mouse monoclonal anti-glial fibrillary acidic protein (GFAP) antibody, mouse monoclonal antiparvalbumin antibody, and the secondary antibodies used here, and isolectin $\mathrm{B}_{4}$ and the immunohistochemical reagents used were purchased from Sigma Chemical Co. (St. Louis, $\mathrm{MO})$. The mouse monoclonal anti-choline acetyltransferase

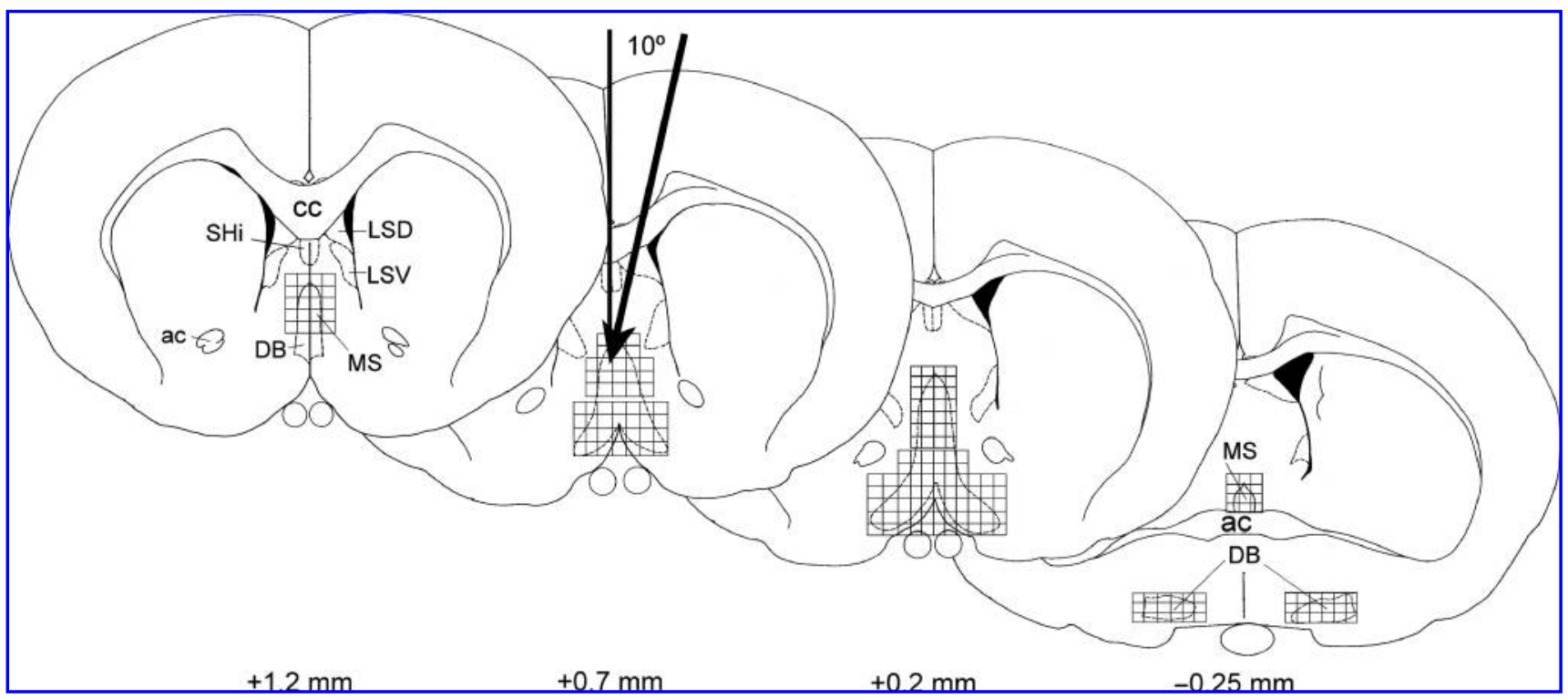

FIG. 1. Schematic drawing of coronal sections showing the four regions of the medial septum-diagonal band of Broca complex where the histologic study was performed. The figures are redrawn from the atlas of Paxinos and Watson (1986), and correspond to levels $+1.2 \mathrm{~mm},+0.7 \mathrm{~mm},+0.2 \mathrm{~mm}$, and $-0.25 \mathrm{~mm}$ relative to the bregma. The arrow shows the cannula placement for the stereotaxic microinjections of AMPA, for which the cannula was inserted at an angle of $10^{\circ}$ from the vertical. Cell counts were made in the areas marked with the grids (ac, anterior commissure; cc, corpus callosum; DB, diagonal band; LSD, lateral septal nucleus dorsal; LSV, lateral septal nucleus ventral; MS, medial septum; SHi, septohippocampal nu). Nomenclature from the atlas of Paxinos and Watson (1986). 
(ChAT) antibody was from Chemicon Inc. (Temecula, CA), and the rabbit monoclonal anti-GC receptor antibody (clone M-20) was from Santa Cruz Biotechnology (Santa Cruz, CA).

\section{Stereotaxic procedure}

The rats were anesthetized with equithesin (a mixture of chloral hydrate and sodium pentobarbitone; $0.3 \mathrm{~mL} / 100 \mathrm{~g}$ body weight IP) and placed on a David Kopf Stereotaxic frame (David Kopf Instruments, Inc., Tujunga, CA) with the incisor bar set at $-3.3 \mathrm{~mm}$. Single intraseptal injections were made through a stainless steel cannula connected by Portex tubing (Sims Medical, Inc., Keene, NH) to a Hamilton syringe activated by an infusion pump (CMA/100; Carnegie Medicin, Stockholm, Sweden). The cannula was inserted at an angle of $10^{\circ}$ from vertical and positioned $0.7 \mathrm{~mm}$ rostral to the bregma and $1.1 \mathrm{~mm}$ lateral and $5.0 \mathrm{~mm}$ ventral from the dura (Paxinos and Watson, 1986) (Fig. 1). The AMPA was dissolved in 50-mM phosphate-buffered saline (PBS; pH 7.4). Injections of $0.5 \mu \mathrm{L}$ were made over a period of $5 \mathrm{~min}$ each, and after completion of the injection the cannula was left in place for an additional $5 \mathrm{~min}$ to allow for passive diffusion and to prevent the spread of the excitotoxin up the cannula track upon removal of the cannula. The cannula was then slowly retracted.

\section{Dose-response study}

Thirty-two animals were divided into eight groups ( $\mathrm{n}=4$ per group). Each rat received a single injection into the medial septum of either PBS, or AMPA at $0.27 \mathrm{nmol}, 1.4 \mathrm{nmol}$, $2.7 \mathrm{nmol}, 5.4 \mathrm{nmol}, 8.1 \mathrm{nmol}$, or $10.8 \mathrm{nmol}$. The remaining untreated rats $(n=4)$ were used as controls. These doses of AMPA were chosen according to results of previous studies (Andrés et al., 2003; Petegnief et al., 1999).

At 2 months after the injection of PBS or AMPA, the rats were anesthetized and perfused transcardially with ice-cold fixative consisting of $0.4 \%(\mathrm{w} / \mathrm{v})$ picric acid and $4 \%(\mathrm{w} / \mathrm{v})$ paraformaldehyde in 0.1 M PBS (pH 7.4). The animals' brains were rapidly removed, post-fixed for $2 \mathrm{~h}$, transferred to $15 \%$ $(\mathrm{w} / \mathrm{v})$ sucrose in PBS, and stored at $4^{\circ} \mathrm{C}$ for 3 days. The brains were then frozen with dry ice and stored at $-80^{\circ} \mathrm{C}$ until use.

\section{Time-course study}

To perform the time-course study of the effects of excitatory injury to the medial septum, we selected the dose of $2.7 \mathrm{nmol}$ AMPA from the dose-response study. We divided 42 rats into two groups, which received either a single injection of PBS $(n=20)$ or AMPA $(n=22)$. The rats were anesthetized and perfused transcardially as previously described at 4 days, 15 days, 1 month, 2 months, or 6 months after injection. At each time point, 4 sham-treated and 4 AMPA-treated animals were killed, except for the final, 6-month time point, at which 4 sham and 6 AMPA-treated rats were used. The animals' brains were rapidly removed, cryoprotected, frozen, and stored at $-80^{\circ} \mathrm{C}$ until used.

\section{Histology and immunohistochemistry}

Serial sections of $12 \mu \mathrm{m}$ each were obtained from each brain along the entire rostrocaudal extension of the medial septumDBB complex. Histological studies were done at four levels of the medial septum to allow full coverage of this area of the brain (the levels $+1.2,+0.7,+0.2$, and $-0.25 \mathrm{~mm}$ relative to the bregma) (Fig. 1). Some sections were processed for Nissl standard staining with cresyl violet. Calcium deposits were visualized by means of staining with alizarin red and counterstaining with fast green (Bernal et al., 2000; Robledo et al., 1999). Histochemistry for isolectin $B_{4}$ was done to identify the microglial reaction as previously described (Colton et al., 1992); some of these sections were counterstained with alizarin red.

Immunohistochemistry was done with the avidin-biotinperoxidase method as previously described (Rodríguez et al., 2004; Ramonet et al., 2004b). Sections were incubated separately at $4^{\circ} \mathrm{C}$ with mouse monoclonal anti-GFAP diluted at $1: 600(\mathrm{v} / \mathrm{v})$ in normal goat serum (NGS; $1: 100 \mathrm{v} / \mathrm{v}$ in $0.01 \mathrm{M}$ PBS; $\mathrm{pH}$ 7.4), mouse monoclonal anti-parvalbumin diluted at $1: 600(\mathrm{v} / \mathrm{v})$ in NGS, mouse monoclonal anti-ChAT diluted at $1: 400(\mathrm{v} / \mathrm{v})$ in NGS, or rabbit monoclonal anti-GC receptor diluted at $1: 750(\mathrm{v} / \mathrm{v})$ in NGS. After incubation with the appropriate secondary antibodies and ExtrAvidin (SigmaAldrich, Inc.), the sections were developed in $0.05 \mathrm{M}$ Tris containing $0.03 \%(\mathrm{w} / \mathrm{v})$ diaminobenzidine and $0.006 \%(\mathrm{v} / \mathrm{v})$ $\mathrm{H}_{2} \mathrm{O}_{2}$. Some sections were counterstained with alizarin red.

The total septal area was measured at the four studied levels of the medial septum on Nissl-stained sections, using a computer-assisted image analysis system (OPTIMAS ${ }^{\circledR}$; BioScan Inc., Washington, DC). The septal area was defined according to Mahy and associates (1995). The laterally adjacent area of the caudate putamen was measured in the same sections to estimate the effects of histological procedures on tissue size, and to correct for variability in individual brain size and tissue shrinkage. Calcified areas were quantified on alizarin red-stained sections as previously described (Petegnief et al., 1999; Robledo et al., 1999). For each sample, color digital images of two different non-counterstained sections were taken with a light microscope, and the total area stained in red was quantified using the OPTIMAS image analysis system. GC receptor density was analyzed densitometrically on black-and-white microscopic images made from GCreceptor immunostained sections (Hwang et al., 2005), also using the OPTIMAS image analysis system. Cell counts were made on parvalbumin and ChAT-immunostained sections. The method of Floderus (Floderus, 1944) was used to correct for the thickness of sections. Because of the difficulty in identifying the border between the medial septum and the DBB, these two areas were separated by drawing a theoretical line at the level of the anterior commissure (Mahy et al., 1995). The area of astroglial reaction was measured on GFAPimmunostained sections.

\section{Three-dimensional reconstruction}

In order to quantify and analyze the precise location of medial septal atrophy and calcium deposit distribution within the medial septum-DBB complex, 3-D reconstruction was done as previously described (Prats-Galino et al., 1999). In brief, using an Olympus BH2 microscope (Olympus, Inc., Tokyo, Japan) with an attached drawing tube, we examined all four of the serial sections of the medial septum and outlined them on paper at a final magnification of $22 \times$. The computer analysis consisted of three steps: (1) digitization of the positions of the calcium deposits and tissue-structure contours was done with a digitizing scanner connected to an image analysis system (Adobe Photoshop 4.0; Adobe 

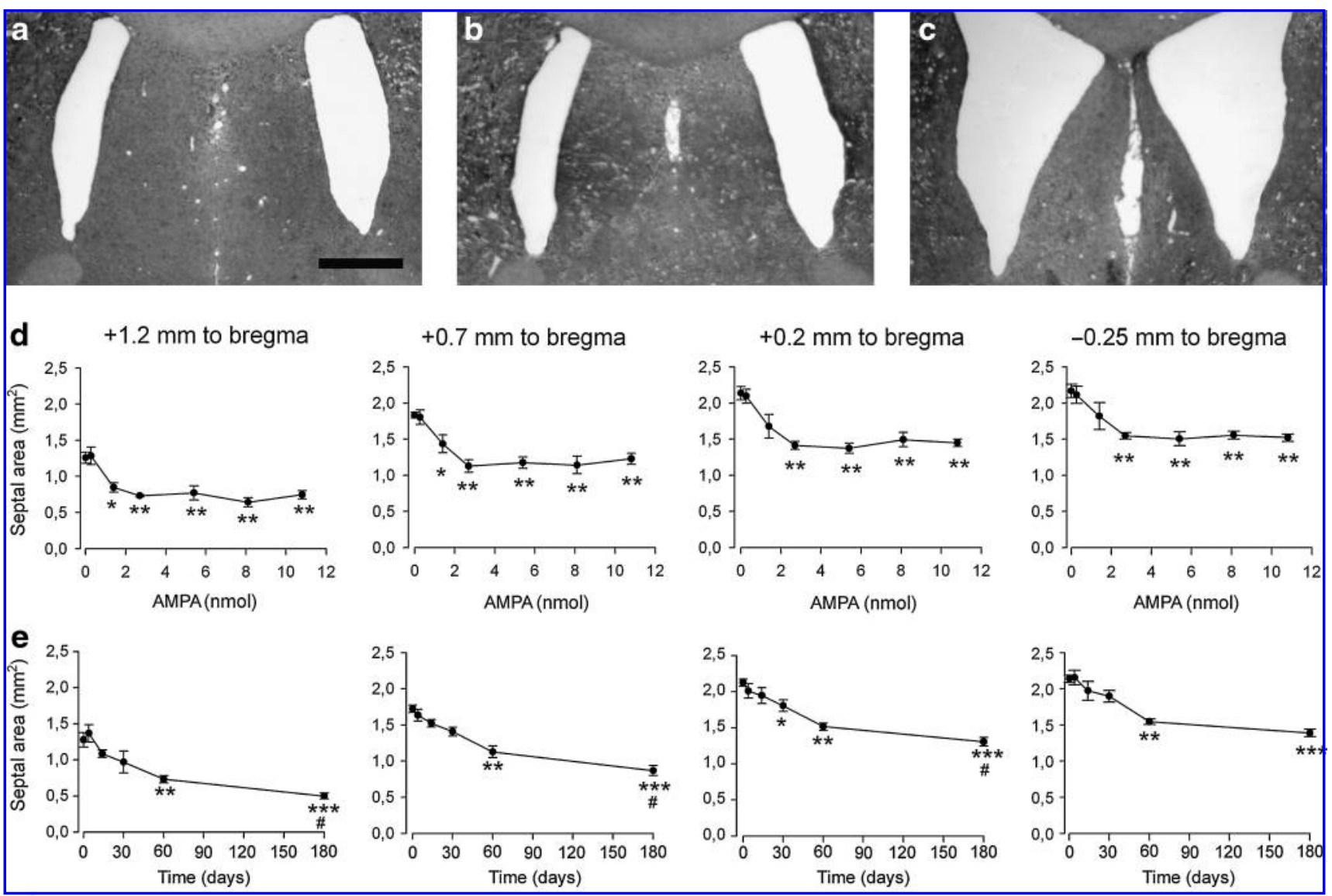

FIG. 2. Effect on septal area size of AMPA-induced lesion in the medial septum. Photomicrographs illustrate the evolution of septal atrophy and show cresyl violet-stained septal areas at the injection level in controls (a), at 15 days (b), and at 6 months (c) after injection of $2.7 \mathrm{nmol}$ of AMPA. Graphs show the quantification of the septal area for all doses of AMPA (d), and for the 6-month course of the study (e) at the four studied levels of the medial septum. Note the different curve shapes, which are quickly saturable for the dose-response study, and far more progressive for the time-course study. For all situations $\mathrm{n}=4$ for both the control and AMPA-treated groups, except for the 6-month time point, at which $\mathrm{n}=4$ for the control group and $\mathrm{n}=6$ for the AMPA-treated group $\left({ }^{*} p<0.05 ;{ }^{* *} p<0.01 ;{ }^{* *} p<0.001\right.$ versus controls; \# $p<0.05$ versus 2 -month lesion on Mann-Whitney $U$ test; scale bar $=250 \mu \mathrm{m})$.

Systems, Inc., San Jose, CA); (2) 3-D surface reconstruction of the serial sections was done by triangulation with software specially developed for this purpose (Prats-Galino et al., 1988); and (3) generation of a shaded 3-D model from the resulting triangulated image by using 3D Studio Max 1.2 software (Kinetix Autodesk, Inc., San Rafael, CA). The original data files were transformed into virtual reality modeling language format and converted with the Crossroads File Format Converter (Crossroads Systems, Inc., Austin, TX) into Autocad drawing exchange format (DXF) (Autodesk, Inc., San Rafael, CA) to be used by 3D Studio Max.

\section{Statistical analysis}

In the time-course study, no age-related change in any of the parameters was observed in control 2- and 8-month-old rats. This was also the case for all of the studies of the shamtreated rats. Because of this, the values recorded for these animals were pooled into a single group and designated the control values. For each parameter, kurtosis and skewness moments were calculated to test for normal distribution of the data. One-way analysis of variance (ANOVA) followed by the post-hoc least significant difference test was used to compare differences between groups. When normality was not reached, the non-parametric Kruskal-Wallis (KW) test followed by the Mann-Whitney $U$ test was used to compare values for all groups. All data are given as mean \pm SEM. Neuronal loss is expressed as a percentage of the control value. Linear regressions were performed to compare the evolution of different parameters. In all cases a value of $p<0.05$ was considered significant. Analyses were done with Statgraphics computer software (STSC Inc., Rockville, MD).

\section{Results}

\section{Dose-response study}

Two months after AMPA injection, a reduction of the entire septal area was found at the four studied levels at all doses of AMPA except for the 0.27-nmol dose (Fig. 2). The progressive reduction in area reached a plateau at $2.7 \mathrm{nmol}$ AMPA, with a value that was $35-40 \%$ of the control value. The KruskalWallis test values for each studied level of the medial septum were: $\mathrm{KW}=22.4, p=0.0010 ; \mathrm{KW}=22.2, p=0.0012$; 


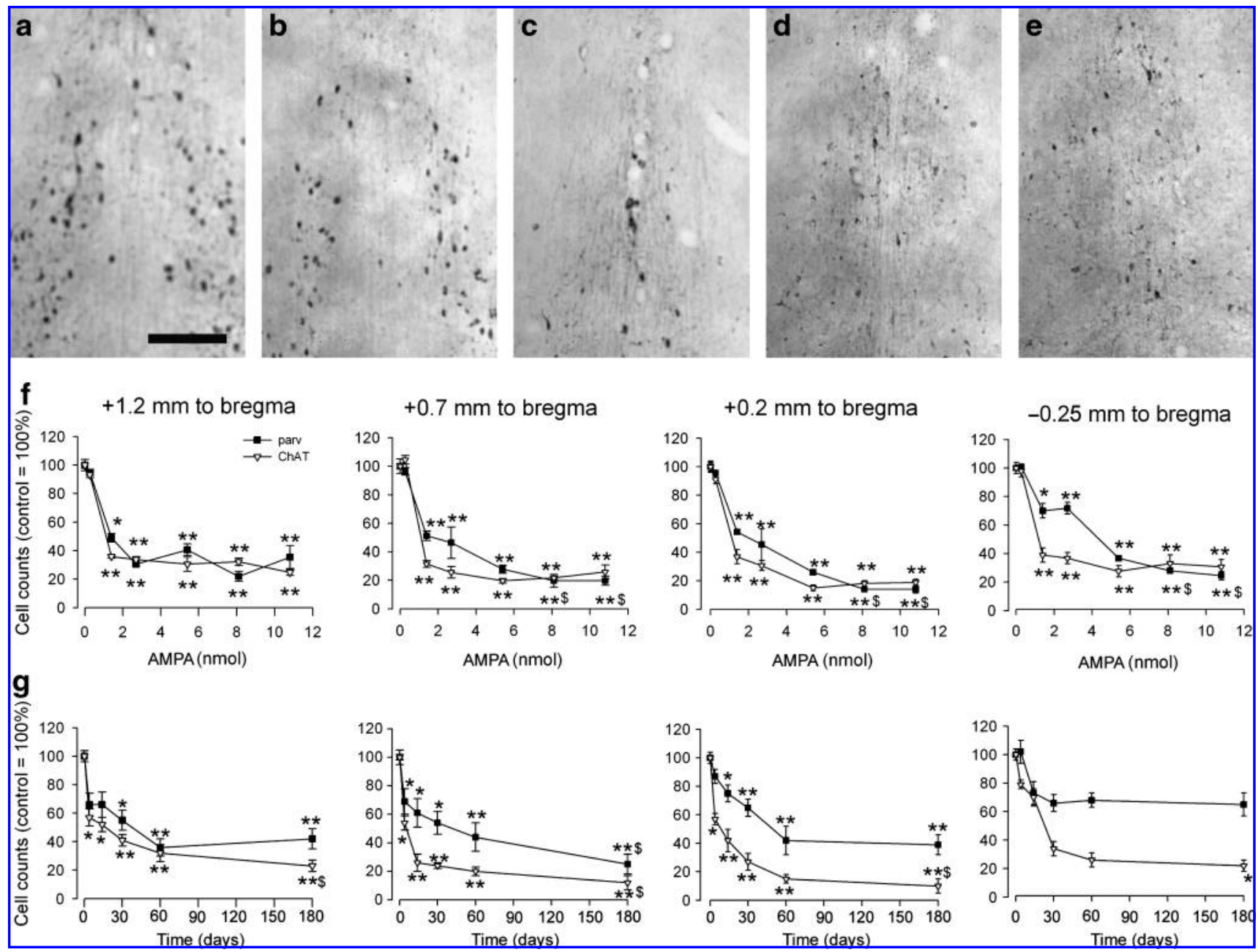

FIG. 3. Characterization of AMPA-induced neuronal loss in the medial septum. Photomicrographs illustrate the neuronal loss at the level of injection, and show ChAT-immunostained sections of control rats (a) and AMPA-lesioned rats at 15 days (b) and 6 months (c) after lesioning, and parvalbumin-immunopositive neurons of control rats (d) and lesioned rats at 2 months (e) after injection of $2.7 \mathrm{nmol}$ of AMPA. Graphs show the quantification of neuronal loss for all AMPA doses (f) and at 6 months (g) of the study at the four studied levels of the medial septum (ChAT, ChAT-immunostained neurons; parv, parvalbumin-immunostained neurons). Note the different sensitivity to AMPA between ChAT-immunopositive and Parvimmunopositive neurons. Note also the different curve shapes, showing that ChAT-immunopositive neurons were rapidly saturable in the dose-response study, and the more progressive curve for the time-course study. For all situations, $\mathrm{n}=4$ for both the control and AMPA-treated groups, except for the 6-month time point, at which $\mathrm{n}=4$ for the control group and $\mathrm{n}=6$ for the AMPA-treated group $\left({ }^{*} p<0.05 ;{ }^{* *} p<0.01\right.$ versus controls; $\$ p<0.05$ versus $2.7 \mathrm{nmol}$ AMPA by Mann-Whitney $U$ test; scale bar $=75 \mu \mathrm{m}$ ).

$\mathrm{KW}=17.7, p=0.0069 ;$ and $\mathrm{KW}=19.7, p=0.0031$, for +1.2 , $+0.7,+0.2$, and $-0.25 \mathrm{~mm}$ relative to the bregma, respectively. When adjacent striatal areas were measured, no differences were found between any of the groups of animals at any of the studied levels (data not shown).

Beginning at $1.4 \mathrm{nmol}$ of AMPA, all four studied septal levels showed a 70-80\% loss of ChAT-immunopositive neurons (Fig. 3) $(\mathrm{KW}=23.41, p=0.0007 ; \mathrm{KW}=23.06, p=0.0008$; $\mathrm{KW}=25.84, p=0.0002$; and $\mathrm{KW}=20.80, p=0.002$, for +1.2 , $+0.7,+0.2$, and $-0.25 \mathrm{~mm}$ relative to the bregma, respectively). ChAT-immunopositive neurons of the DBB were decreased only at the level of AMPA injection, owing to a slight loss of neurons in the vertical limb. When the size of areas of ChAT-immunopositive neurons in the medial septum was measured directly on photomicrographs, no difference was found between controls (mean diameter: $18 \pm 2 \mu \mathrm{m} ; \mathrm{n}=40$ ) and lesioned animals (mean diameter: $17 \pm 2 \mu \mathrm{m} ; \mathrm{n}=60$ ).

GABAergic neurons were also sensitive to AMPA. At the injection level, the percentage of parvalbuminimmunopositive neurons was decreased by $55-80 \%$ with increasing doses of AMPA $(\mathrm{KW}=24.13, p=0.0005 ; \mathrm{KW}=23.62$, $p=0.0006 ; \quad \mathrm{KW}=24.18, \quad p=0.0005 ; \quad$ and $\mathrm{KW}=24.13$, $p=0.0005$, for $+1.2,+0.7,+0.2$, and $-0.25 \mathrm{~mm}$ relative to the bregma, respectively). The most severe GABAergic neuronal loss was reached at a dose of $5.4 \mathrm{nmol}$ AMPA. Direct measurement of neuronal body size showed no difference between controls (mean diameter: $20 \pm 4 \mu \mathrm{m} ; \mathrm{n}=40$ ) and lesioned animals (mean diameter: $19 \pm 3 \mu \mathrm{m} ; \mathrm{n}=30$ ). 

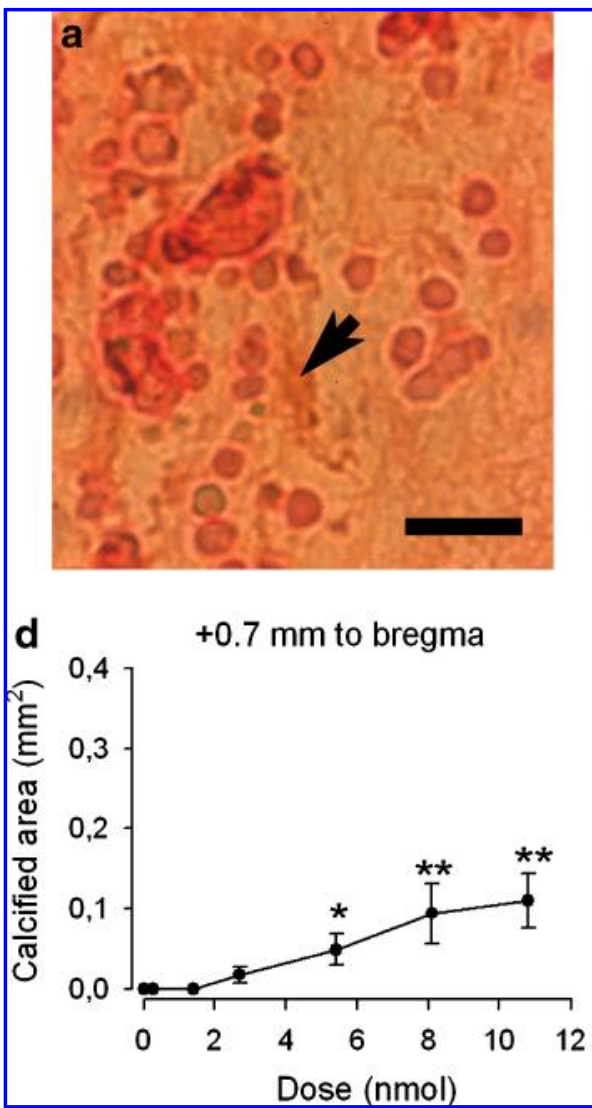
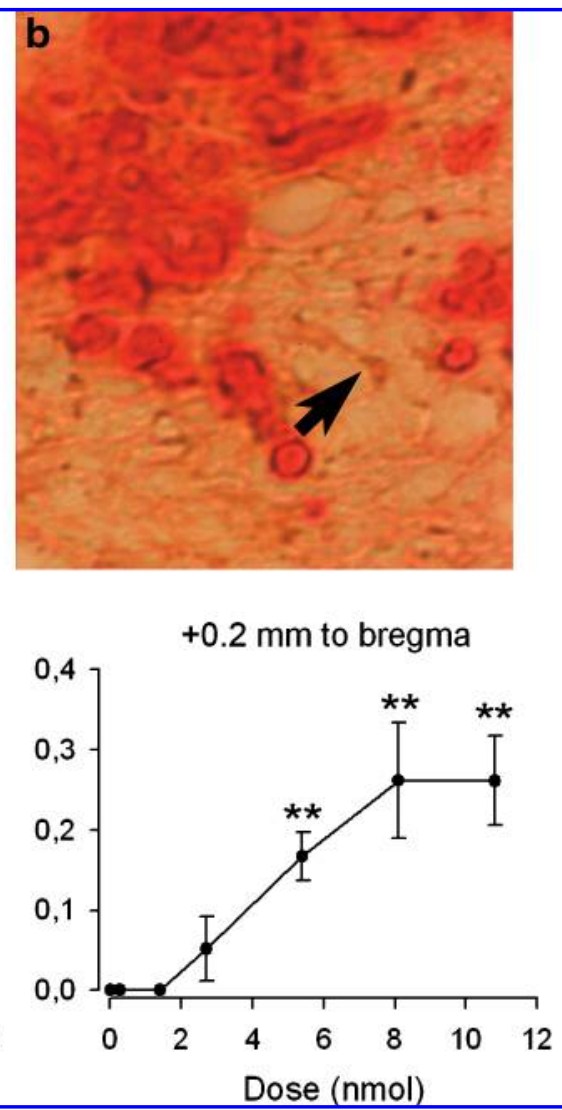
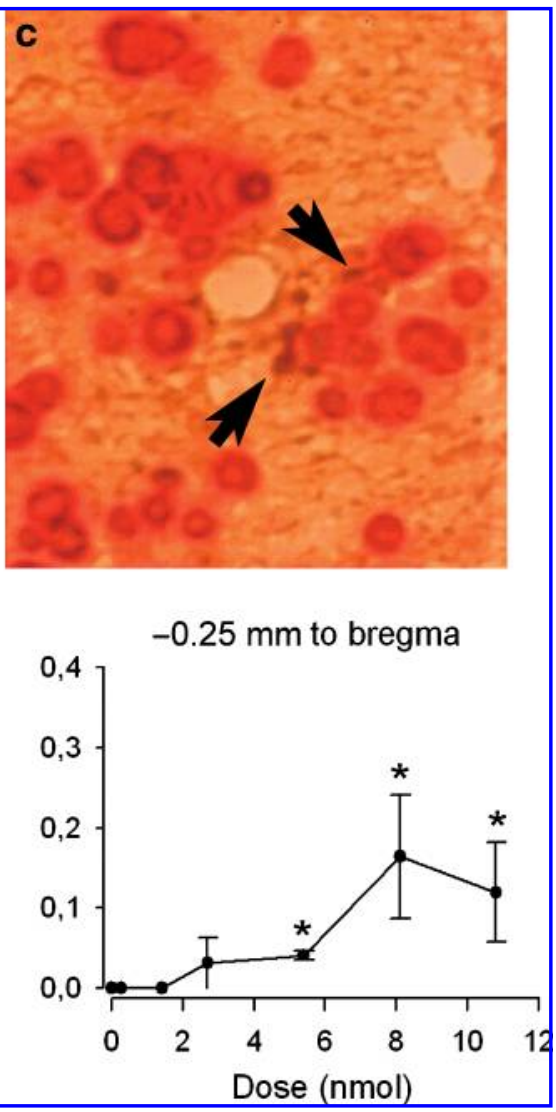

FIG. 4. Analysis of calcification induced by AMPA in the medial septum. Photomicrographs of (a) parvalbuminimmunostained neurons (arrow), (b) GFAP-immunostained astrocytes (arrow), and (c) isolectin $\mathrm{B}_{4}$-stained microglia in sections counterstained with alizarin red. Note the calcium deposits localized at sites where GABAergic neurons are more abundant, and the close relationship between calcium deposits and reactive microcytes (arrows in c). The dose of AMPA was $8.1 \mathrm{nmol}$. (d) Graphs showing the quantification of calcium deposits at the three septal levels that exhibited calcification. In all cases $\mathrm{n}=4$ for both the control and AMPA-treated groups $\left({ }^{*} p<0.05 ;{ }^{* *} p<0.01\right.$ versus controls on Mann-Whitney $U$ test; scale bar $=40 \mu \mathrm{m})$. (Color image is available online at www.liebertpub.com/jon)

With the exception of the most rostral level of the medial septum $(+1.2 \mathrm{~mm}$ relative to the bregma), calcium deposits were found only at doses of AMPA higher than $2.7 \mathrm{nmol}$ $(\mathrm{KW}=16.7, p=0.0021$ at $+0.7 \mathrm{~mm}$ relative to the bregma; $\mathrm{KW}=17.2, p=0.0042$ at $+0.2 \mathrm{~mm}$ relative to the bregma; and $\mathrm{KW}=10.9, p=0.026$ at $-0.25 \mathrm{~mm}$ relative to the bregma). The 2.7-nmol dose of AMPA induced calcification in the medial septum in only half of the animals. Although showing a tendency to increase with increasing AMPA dose, this calcification process was not dose-dependent (Fig. 4). Localized within the GABAergic neuronal area, these precipitates appeared mainly in the center of the medial septum, and were less common in the ventral region of the DBB. The precipitates showed no relationship to blood vessels or to the injectionneedle scar.

No astroglial reaction was detected by GFAP immunohistochemistry in the septal area. Only the needle scar showed a slight increase in astroglial immunostaining. At all doses of AMPA above $2.7 \mathrm{nmol}$, the astrocytes showed a different morphology, characterized by smaller size and fewer cellular processes in the lesioned area than in the lateral septum or the medial septum of control rats (Fig. 5). Histochemistry for isolectin $\mathrm{B}_{4}$-stained activated microglia were positive only in
AMPA-treated animals that showed calcium deposits. Alizarin red counterstaining showed reactive microcytes associated with calcium deposits in the center of the medial septum-DBB complex.

\section{Time-course study}

The 2.7-nmol dose of AMPA was selected for the doseresponse study because of its efficacy in producing an intermediate state of septal lesioning. The progressive decrease in septal area at the four studied levels $(\mathrm{KW}=21.9, p=0.0005$; $\mathrm{KW}=23.3, p=0.0003 ; \mathrm{KW}=21.4, p=0.0007$; and $\mathrm{KW}=22.3$, $p=0.0005$, for $+1.2,+0.7,+0.2$, and $-0.25 \mathrm{~mm}$ relative to the bregma, respectively) reached significance at 2 months after AMPA injection (at 35\% of the control value), and was still increasing at 6 months after injection (Fig. 2). When adjacent striatal areas were measured, no difference was again found between any groups of animals at any of the studied levels (data not shown).

At 4 days after injection of the 2.7-nmol dose of AMPA, a $40 \%$ loss of ChAT-immunoreactive neurons was observed in the medial septum $(\mathrm{KW}=21.6, p=0.0006 ; \mathrm{KW}=17.7$, $p=0.0014 ; \mathrm{KW}=22.5, p=0.0004 ;$ and $\mathrm{KW}=21.7, p=0.0008$, 

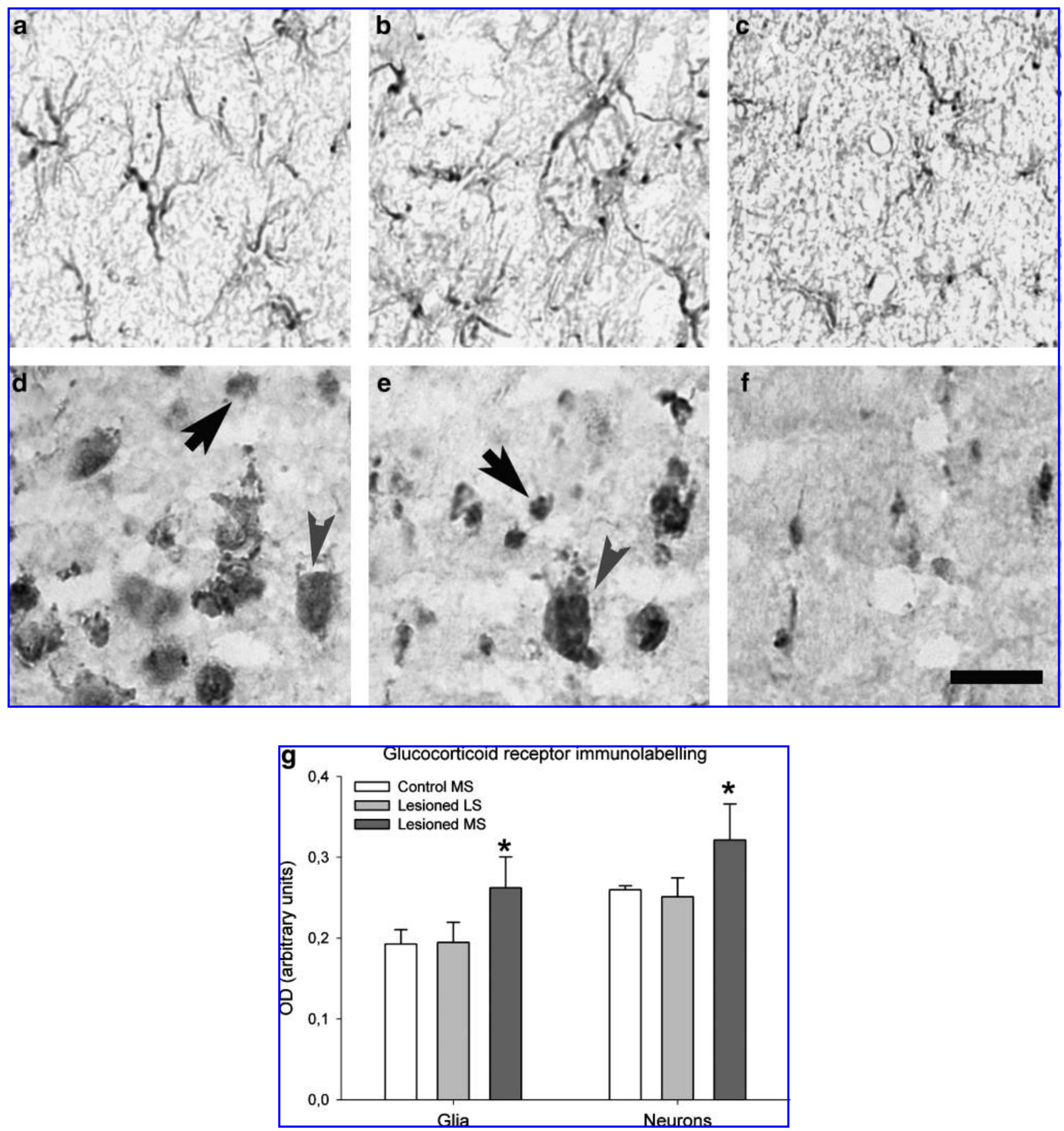

FIG. 5. Effects of AMPA on septal astrocytes and GC receptor distribution. Photomicrographs show astrocyte morphology $(\mathbf{a}-\mathbf{c})$ and GC receptor distribution $(\mathbf{d}-\mathbf{f})$ in the medial septa of control rats and rats treated with $2.7 \mathrm{nmol}$ AMPA at 2 months after lesioning. GFAP immunohistochemistry in medial septum of control rats (a), lateral septum of lesioned rats (b), and medial septum of lesioned rats (c). Note the loss of processes and cellular shrinkage in c as compared with a and b. GC immunohistochemistry in control rats (d) and lesioned rats (e). There is evidence of increased labeling in surviving neurons (arrowheads) and astrocytes (arrows). Astrocytes in the lesioned area of the medial septum that lacked surviving neurons (f) also showed increased GC immunoreactivity. (g) Graph of the quantification of GC immunoreactivity in cells from the septal area at the level of injection of AMPA. In all cases $n=4$ for both the control and AMPA-treated groups (LS, lateral septum; MS, medial septum; OD, optical density; ${ }^{*} p<0.05$ versus controls by Mann-Whitney $U$ test; scale bar $\left.=30 \mu \mathrm{m}\right)$. 
Table 1. Linear Regression Analysis of Medial Septal Lesion at +0.2 mm to the Bregma

\begin{tabular}{llll}
\hline & \multicolumn{1}{c}{ Septal area } & ChAT counts & Parvalbumin counts \\
\hline $\begin{array}{l}\text { Dose-response } \\
\text { ChAT counts }\end{array}$ & $\mathrm{r}^{2}=0.61, p=0.0001$ & & \\
$\quad \begin{array}{l}\text { Parvalbumin counts } \\
\text { Calcified area }\end{array}$ & $\mathrm{r}^{2}=0.52, p=0.0001$ & $\mathrm{r}^{2}=0.82, p<0.00001$ & $\mathrm{r}^{2}=0.46, p<0.001$ \\
$\begin{array}{l}\text { Time-course } \\
\quad \text { ChAT counts }\end{array}$ & $\mathrm{r}^{2}=0.29, p=0.0017$ & & \\
Parvalbumin counts & $\mathrm{r}^{2}=0.45, p=0.0001$ & $\mathrm{r}^{2}=0.57, p<0.00001$ & \\
\hline
\end{tabular}

ChAT, choline acetyltransferase.

for $+1.2,+0.7,+0.2$, and $-0.25 \mathrm{~mm}$ relative to the bregma, respectively), and increased progressively to reach $85 \%$ after 6 months (Fig. 3). A similar progression was also seen in the vertical limb of the DBB. Parvalbumin-immunostained neurons were reduced in number only at the three anterior septal levels $(\mathrm{KW}=15.5, p=0.0082 ; \mathrm{KW}=18.3, p=0.0025$; $\mathrm{KW}=21.1, p=0.0008$, for $+1.2,+0.7$, and $+0.2 \mathrm{~mm}$ relative to the bregma, respectively), with an insignificant reduction at $-0.25 \mathrm{~mm}$ to the bregma. At 4 days the reduction reached $35 \%$, but thereafter progressed only for 2 months (Fig. 3). ChAT- or parvalbumin-immunostained neurons of lesioned rats were of the same size as those of the controls.

No calcium deposits were found in the septal area of any rat at any time. At 4 days after the injection of PBS, immunostaining for GFAP showed a small area of astrogliosis $\left(0.27 \pm 0.02 \mu \mathrm{m}^{2}\right)$ localized at the injection site. At this same 4-day time point, AMPA injection had induced marked astrogliosis in the lateral and medial septum and in the vertical limb of the DBB throughout the rostrocaudal extension of the latter structure $\left(1.41 \pm 0.08 \mu \mathrm{m}^{2}\right.$ at the injection level). This astrogliosis, although still present at 15 days after AMPA injection, was reduced in intensity and extent $\left(0.81 \pm 0.06 \mu \mathrm{m}^{2}\right.$ at the injection level), and was no longer detectable at 1 month after lesioning. At longer intervals, astrocytes in the lesioned area of the medial septum showed an abnormal morphology, with reduced size and fewer cellular processes than control cells (Fig. 5). In no animal did staining for isolectin $\mathrm{B}_{4}$ show a microglial reaction in the septal area.

\section{Glucocorticoid receptors}

Immunohistochemistry for GC receptors showed a widespread distribution of these receptors in the medial septumDBB complex, lateral septal nuclei, and adjacent areas of control animals. Staining specific for GC receptors was present in the cytoplasm and nuclei of neurons and glial cells (Fig. 5), but was always stronger in the cells' nuclei. At 2 months after the injection of $2.7 \mathrm{nmol}$ of AMPA, increased GC receptor immunoreactivity in the lesioned area of the medial septum $(\mathrm{KW}=13.187 ; p=0.021)$ was observed, mainly in the nuclei of astrocytes (a 36\% increase), and surviving neurons (a $24 \%$ increase). No increase was observed in the DBB, lateral septum, or caudate putamen.

\section{Correlations and 3-D reconstruction}

Statistical correlation studies done to estimate the relationship between septal atrophy, neuronal loss, and calcification gave similar results at all four studied levels of the medial septum (Table 1). In the dose-response and timecourse studies, the correlation coefficient for the number of ChAT-immunostained neurons with neuronal atrophy was greater than that for the number of parvalbuminimmunostained neurons and atrophy. The greatest correlation was found between cholinergic and GABAergic neuronal loss. In the dose-response study, only the calcified area showed a positive correlation with GABAergic neuronal loss.

$3-\mathrm{D}$ reconstruction clearly showed the contribution of lateral septal nuclei to atrophy of the septal area (Fig. 6) at 2 months after injection of the 2.7-nmol dose of AMPA, and also showed the increase in this atrophy with both increasing AMPA concentration and time after lesioning. No specific atrophy was detected in the DBB. In this analysis, increasing time after lesioning showed a greater effect on septal atrophy than did AMPA concentration. Neuronal loss in the medial septum-DBB complex, which was restricted to the medial septum and a small part of the vertical limb of the DBB, increased with AMPA concentration, but not with time after lesioning. AMPA-induced calcification was observed at the center of the medial septum and ventral region of the DBB, which are rich in GABAergic neurons. The calcification was limited to the lesioned area and located caudally to the injection site.

\section{Discussion}

This study demonstrates that acute AMPA-induced lesioning of the medial septum triggers a long-term neurodegenerative process characterized by progressive septal atrophy and a loss of cholinergic and GABAergic neurons, as

FIG. 6. 3-D reconstructions of the rat medial septum-DBB complex and adjacent structures. Illustrative representation of a 3 -D reconstruction of the septal zone (a), medial septum (b), and lesioned medial septum (c) of one control and three lesioned rats at different post-lesion time-points and AMPA doses. Each structure has a different color: lateral ventricles are shown in red, medial septum in blue, anterior commissure in green, lesioned medial septum in yellow, and calcification in pink. The observer is at the rostral (a) and rostrolateral (b and c) sides of the reconstruction. To allow for visualization of lesions and calcification inside the medial septum, structures in $b$ and $c$ are presented as triangular facets (r, right; l, left; scale bar $=1 \mathrm{~mm}$ ). (Color image is available online at www.liebertpub.com/jon) 


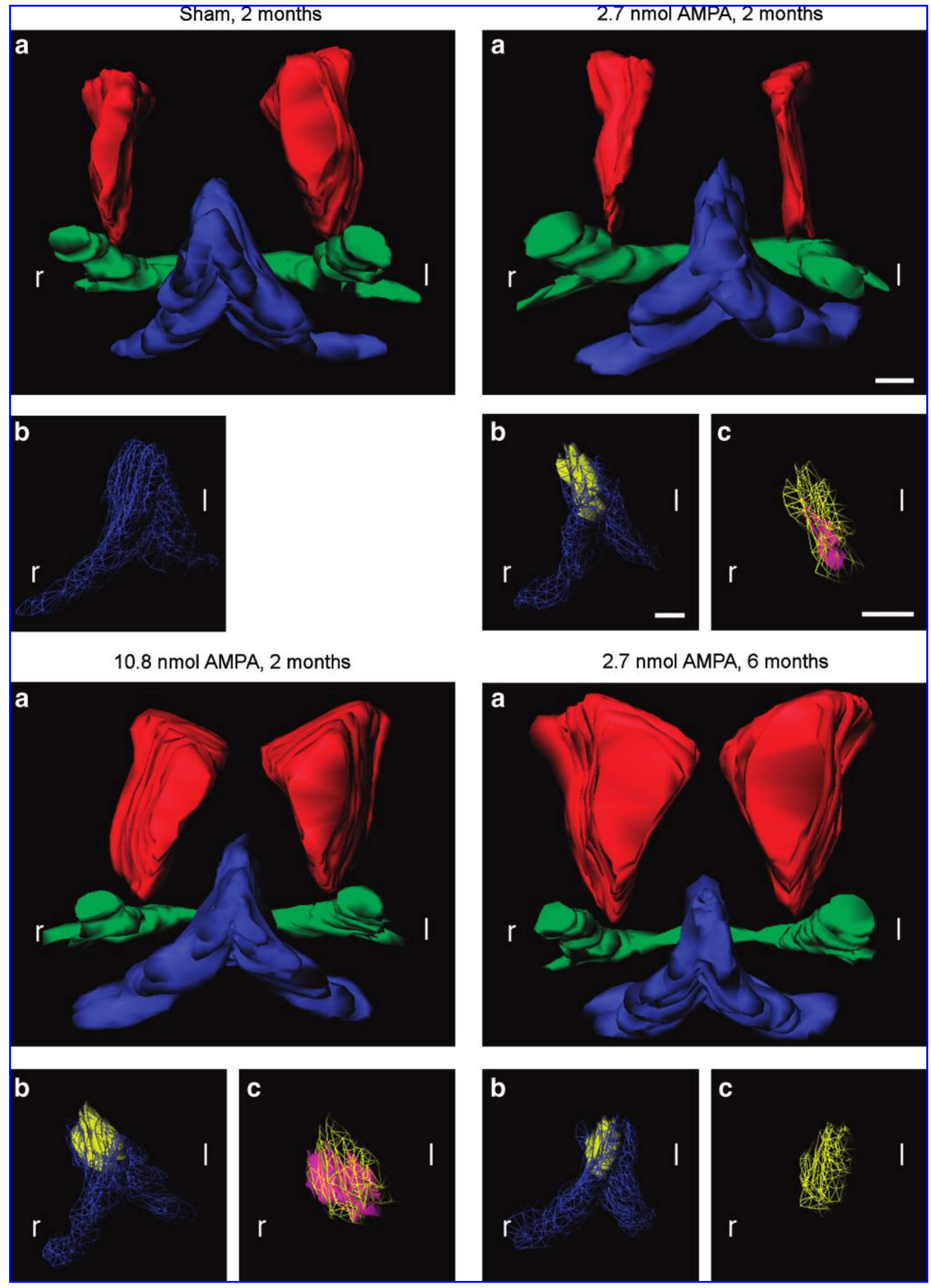


well as increased GC receptor density in the absence of a astroglial or microglial reaction. The septal atrophy, cholinergic and GABAergic neuronal loss, and calcium precipitation observed in the dose-response study done with AMPA imply the activation of a mechanism that differs from that in the ongoing neurodegenerative process.

Compared to the acute injury induced by AMPA, whose effects reached a plateau at about $5.4 \mathrm{nmol}$ of AMPA, the longterm evolution of the lesion induced by this neurotoxin was characterized by increasing and continuing atrophy and cholinergic neuronal loss in the absence of astrogliosis throughout the 6-month duration of our study. Previous studies done with quisqualic or ibotenic acid under similar conditions, found a similarly severe atrophy of the medial septum after a 1-year period (Ramani et al., 2006; Yoshiyama et al., 2005; Leutgeb and Mizumori, 1999; Mahy et al., 1995). Notwithstanding the heterogeneity in anatomic organization and neuronal composition of the medial septum (Kiss et al., 1990), our data showed a similar atrophy throughout its entire structure. 3-D reconstruction and the astrogliosis observed within the first 15 days after lesioning indicate an initial injury to the lateral nuclei from the microinjection of AMPA into the medial septum. This is no surprise given the high glutamate receptor density of the lateral septal nuclei (Le Jeune et al., 1996; Stewart and Vanderwolf, 1987). Although the progressive loss of cholinergic neurons observed in our study correlates with atrophy, it cannot be associated with neuronal shrinkage, since no reduction in the size of cholinergic and GABAergic neuronal soma was found. However, other factors, such as lesioning of the lateral septum, absence of an astroglial reaction, and reduction in numbers of dendritic processes, may all contribute to a reduction in septal area. Prolonged exposure to high concentrations of GCs may exacerbate the adverse consequences of chronic brain insults (Sorrells and Sapolsky, 2007). Thus for example, shrinkage of astrocytes and a reduction in the numbers of their processes, as found at 2 months after injection of AMPA in our study, may result from a reduced uptake of glucose consequent to an increased density of GC receptors and loss of synaptic contacts (Tata et al., 2006).

Calcification develops in most areas of the rat brain following the in-vivo microinjection of several excitotoxins, and also develops in humans after acute brain injury (Ramonet et al., 2006; Casanova and Araque, 2003; Rodríguez et al., 2001; Herrmann et al., 1998; Ansari et al., 1990). The pattern of calcification in each area of the brain reflects its sensitivity to injury (Lammie et al., 2005; Rodríguez et al., 2004; Bernal et al., 2000; Mahy et al., 1995). The medial septum, which is more resistant to AMPA-induced excitotoxicity than are the hippocampus, globus pallidus, and cerebral cortex (Rodríguez et al., 2000), developed calcification only at the highest concentrations of AMPA, but not over a long time interval after lesioning. This is consistent with calcification in the human brain induced by perinatal ischemia and hypoxia, which has been found to be directly related to the intensity of an insult, but not to the time after its occurrence (Rodríguez et al., 2001; Ansari et al., 1990).

Among the factors responsible for the resistance of the medial septum to calcification and excitotoxicity are glutamate receptors, calcium-binding proteins, a low level of energy availability, astrocytes, and GABAergic neurons, all of which are directly involved in the brain-calcification process
(Ramonet et al., 2006; Rodríguez et al., 2004). A reason for this is that the medial septum, compared with other areas such as the hippocampus, has fewer AMPA binding sites with a similar pharmacologic specificity (Le Jeune et al., 1996; Nicolle et al., 1996), and the same is true for metabotropic glutamate receptors of type I (Hollmann and Heinemann, 1994). The parvalbumin expressed in GABAergic neurons rapidly retains large amounts of free calcium (Rodríguez et al., 2004), and thus helps to reduce calcium mobility and calcification. This facilitates the marked resistance to injury of astrocytes, reduced formation of lactate as an energy source, and absence of microgliosis observed in the medial septum (Rodríguez et al., 2004; Ramonet et al., 2004a; Ramonet et al., 2002). The lack of neuronal calcification seen at 6 months after injection of the 2.7-nmol dose of AMPA qualifies calcification as an acute process. A microglial reaction, such as that observed in this study, is a direct phagocytic and inflammatory response to the formation of calcium deposits (Rodríguez et al., 2004; Saura et al., 1995).

In contrast to the dose-response data for the injection of AMPA that were obtained in our study, septal atrophy and cholinergic neuronal loss progressed with time without associated astrogliosis or microgliosis. From 15 days on, the effects of acute injury are counteracted (Sapolsky, 2001), but this is not true for the underlying long-term process that leads to tissue atrophy and cholinergic neuronal loss, as is also observed in Alzheimer's disease (Mamelak, 2007). Astrocyte dysfunction is a key factor in the pathogenesis of human neurologic disorders (Seifert et al., 2006), and in the cognitive impairment of aged rats (Andrés et al., 2000). In the case of the chronic lesion, the combined atrophy of astrocytes and lack of astrogliosis suggest a maladaptive response that may be a cause of the ongoing pathologic process.

In sum, the AMPA-induced septal neurodegeneration observed in our study involves specific mechanisms that protect against GABAergic neuronal loss and calcification. This implies astroglial dysfunction and probably participation by GC signaling pathways in this neurodegeneration. These features, absent with increasing AMPA concentration, would then be parameters of chronic damage underlying hippocampal dysfunction. Accordingly, our data showing differences between the acute effects of a graded excitotoxic lesion and its effects over an extended course of time may help explain the differences observed in rat behavior with acute as opposed to chronic injury to the medial septum. Excitotoxic lesioning of the medial septum reproduces the memory impairments observed with aging in the rat (Leutgeb and Mizumori, 1999; Gallagher et al., 1993). In this setting, the best results are not obtained after an intense cholinergic injury to the medial septum (Waite et al., 1994), but by modifying the relative strengths of intrahippocampal inhibitory and excitatory connections (Leutgeb and Mizumori, 1999).

\section{Conclusion}

In conclusion, the study described here demonstrates that the effects that we observed in the medial septum in response to graded excitotoxic insults reached a plateau with time. In contrast, the long-term neurodegenerative process triggered by these insults develops with specific pathogenic mechanisms that increase septal damage with the passage of time. These differences in sensitivity of the medial septum to acute 
and chronic damage underlie the involvement of different mechanisms activated by such damage. This indicates that paradigms for the short-term effects of injury to the brain are inappropriate for elucidating the pathogenic mechanisms of long-term neurodegenerative processes.

\section{Acknowledgments}

This work was supported by grant SAF2005-04314 from the Ministerio de Educación y Ciencia, and grant CIBERNED de enfermedades neurodegenerativas from the Ministerio de Sanidad y Consumo of Spain, and by grant 2005SGR00609 from the Generalitat de Catalunya, Spain.

\section{Author Disclosure Statement}

No conflicting financial interests exist.

\section{References}

Andrés, N., Malpesa, Y., Rodríguez, M.J., and Mahy, N. (2003). Low sensitivity of retina to AMPA-induced calcification. J. Neurosci. Res. 72, 543-548.

Andrés, N., Rodríguez, M.J., Andrade, C., Rowe, W., Quirion, R., and Mahy, N. (2000). Increase in AMPA receptors in aged memory-impaired rats is not associated with increase in monoamine oxidase B levels. Neuroscience 101, 807-810.

Ansari, M.Q., Chincanchan, C.A., and Armstrong, D.L. (1990). Brain calcification in hypoxic-ischemic lesions: an autopsy review. Pediatr. Neurol. 6, 94-101.

Arendt, T., Bigl, V., Tennsted, A., and Arendt, A. (1985). Neuronal loss in different parts of the nucleus basalis is related to neuritic plaque formation in cortical target areas in Alzheimer's disease. Neuroscience 14, 1-14.

Arundine, M., and Tymianski, M. (2004). Molecular mechanisms of glutamate-dependent neurodegeneration in ischemia and traumatic brain injury. Cell Mol. Life Sci. 61, 657-668.

Baptiste, D.C., and Fehlings, M.G. (2006). Pharmacological approaches to repair the injured spinal cord. J. Neurotrauma 23, 318-334.

Bernal, F., Saura, J., Ojuel, J., and Mahy, N. (2000). Differential vulnerability of hippocampus, basal ganglia and prefrontal cortex to long-term NMDA excitotoxicity. Exp. Neurol. 161, 686-695.

Brandner, C.S.K. (1988). Septal lesions impair the acquisition of a cued place navigation task: attentional of memory deficit? Neurobiol. Learn Mem. 69, 106-125.

Carvey, P.M., Punati, A., and Newman, M.B. (2006). Progressive dopamine neuron loss in Parkinson's disease: The multiple hit hypothesis. Cell Transplant 15, 239-250.

Casanova, M.F., and Araque, J.M. (2003). Mineralization of the basal ganglia: implications for neuropsychiatry, pathology and neuroimaging. Psychiatry Res. 121, 59-87.

Colton, C.A., Abel, C., Patchett, J., Keri, J., and Yao, J. (1992). Lectin staining of cultured CNS microglia. I. Histochem. $\mathrm{Cy}-$ tochem. 40, 505-512.

Etienne, P., Robitaille, Y., Wood, P., Gauthier, S., Nair, N.P., and Quirion, R. (1986). Nucleus basalis neuronal loss, neuritic plaques and choline acetyltransferase activity in advanced Alzheimer's disease. Neuroscience 19, 1279-1291.

Floderus, S. (1944). Untersuchungen über den Bau der Menschlichen Hypophise mit besonderer Berucksichtigung der qualitativen mikromorphologischen Verhältnisse. Acta Pathol. Microbiol. Scand. 53, 1-276.
Gallagher, M., Burwell, R., and Burchinal, M. (1993). Severity of spatial learning impairment in aging: development of a learning index for performance in the Morris water maze. Behav. Neurosci. 107, 618-626.

Ginsberg, S.D., Cailliau-Portera, C., and Martin, L.J. (1999). Fimbria-fornix transection and excitotoxicity produce similar neurodegeneration in the septum. Neuroscience 88, 10591071.

Hazell, A.S. (2007). Excitotoxic mechanisms in stroke: An update of concepts and treatment strategies. Neurochem. Int. 50, 941953.

Herrmann, G., Stünitz, H., and Nitsch, C. (1998). Composition of ibotenic acid-induced calcifications in rat substantia nigra. Brain Res. 786, 205-214.

Hollmann, M., and Heinemann, S. (1994). Cloned glutamate receptors. Annu. Rev. Neurosci. 17, 31-108.

Hwang, I.K., Lee, Y.B., Yoo, K.Y., Kang, T.C., Kim, D.W., Moon, W.K., Kim, D.W., Oh, Y.S., Sohn, H.S., and Won, M.H. (2005). Seizure-induced changes of mineralocorticoid and glucocorticoid receptors in the hippocampus in seizure sensitive gerbils. Neurosci Res 2005;53:14-24.

Kiss, J., Patel, A.J., Bainbridge, K.G., and Freund, T.F. (1990). Topographical localization of neurons containing parvalbumin and choline acetyltransferase in the medial septumdiagonal band region of the rat. Neuroscience 36, 61-72.

Koistinaho, M., and Koistinaho, J. (2005). Interactions between Alzheimer's disease and cerebral ischemia-focus on inflammation. Brain Res. Brain Res. Rev. 48, 240-250.

Lammie, G.A., Kelly, P.A.T., Baird, J.D., Smith, W., Chatterjee, S., Frier, B.M., and Strachan, M.W. (2005). Basal ganglia calcification in BB/E rats with diabetes. J. Clin. Neurosci. 12, 49-53.

Le Jeune, H., Cécyre, D., Rowe, W., Meaney, M.J., and Quirion, R. (1996). Ionotropic glutamate receptor subtypes in the aged memory-impaired and unimpaired Long-Evans rat. Neuroscience 74, 349-363.

Leutgeb, S., and Mizumori, S.J.Y. (1999). Excitotoxic septal lesions result in spatial memory deficits and altered flexibility of hippocampal single-unit representations. J. Neurosci. 19, 6661-6672.

Mahy, N., Bendahan, G., Boatell, M.L., Bjelke, B., Tinner, B., Olson, L., and Fuxe, K. (1995). Differential brain area vulnerability to long-term subcortical excitotoxic lesions. Neuroscience $65,15-25$.

Mamelak, M. (2007). Alzheimer's disease, oxidative stress and gammahydroxybutyrate. Neurobiol. Aging 28, 1340-1360.

McEwen, B.S. (1992). Re-examination of the glucocorticoid hypothesis of stress and aging. Prog. Brain Res. 93, 365-381.

Miele, V.J., Bailes, J.E., Cantu, R.C., and Rabb, C.H. (2006). Subdural hematomas in boxing: the spectrum of consequences. Neurosurg. Focus 21, E10.

Nicolle, M.M., Bizon, J.L., and Gallagher, M. (1996). In vitro autoradiography of ionotropic glutamate receptors in hippocampus and striatum of aged Long-Evans rats: relationship to spatial learning. Neuroscience 74, 741-756.

Paxinos, G., and Watson, C. (1986). The Rat Brain in Stereotaxic Coordinates. Academic Press: Sydney.

Petegnief, V., Saura, J., Dewar, D., Cummins, D.J., Dragunow, M., and Mahy, N. (1999). Long-term effects of $\alpha$-amino3-hydroxy-5-methyl-4-isoxazole propionate and 6-nitro-7sulphamoylbenzo(f)quinoxaline-2,3-dione in the rat basal ganglia: calcification, changes in glutamate receptors and glial reactions. Neuroscience 94, 105-115.

Pluta, R. (2004). Alzheimer lesions after ischemia-reperfusion brain injury. Folia Neuropathol. 42, 181-186. 
Prats-Galino, A., Costa-Llobet, C., Arroyo-Guijarro, J., and RuanoGil, D. (1988). The facial motor nucleus of the dog. I. Cytoarchitectonic subdivisions and cytology. Acta Anat. 132, 276-279.

Prats-Galino, A., Puigdellívol-Sánchez, A., Ruano-Gil, D., and Molander, C. (1999). Representations of hindlimb digits in rat dorsal root ganglia. J. Comp. Neurol. 408, 137-145.

Ramani, A., Jensen, J.H., and Helpern, J.A. (2006). Quantitative MR imaging in Alzheimer disease. Radiology 241, 26-44.

Ramonet, D, De Yebra L, Fredriksson K, Bernal F, Ribalta T, Mahy N. (2006). Similar calcification process in acute and chronic human brain pathologies. J. Neurosci. Res. 83, 147-156.

Ramonet, D., Pugliese, M., Rodríguez, M.J., De Yebra, L., Andrade, C., Adroer, R., Ribalata, T., Mascort, J., Mahy, N. (2002). Calcium precipitation in acute and chronic brain diseases. J. Physiol. (Paris) 96, 307-312.

Ramonet, D., Rodríguez, M.J., Fredriksson, K., Bernal, F., and Mahy, N. (2004a). In vivo neuroprotective adaptation of the glutamate/glutamine cycle to neuronal death. Hippocampus 14, 586-594.

Ramonet, D., Rodríguez, M.J., Pugliese, M., and Mahy, N. (2004b). Putative glucosensing property in rat and human activated microglia. Neurobiol. Dis. 17, 1-9.

Rao, S.D., and Weiss, J.H. (2004). Excitotoxic and oxidative crosstalk between motor neurons and glia in ALS pathogenesis. Trends Neurosci. 27, 17-23.

Robledo, P., Ursu, G., and Mahy, N. (1999). Effects of adenosine and gamma-aminobutyric acid A receptor antagonists on $\mathrm{N}$ methyl-D-aspartate induced neurotoxicity in the rat hippocampus. Hippocampus 9, 527-533.

Rodríguez, M.J., Bernal, F., Andrés, N., Malpesa, Y., and Mahy, N. (2000); Excitatory amino acids and neurodegeneration: A hypothetical role of calcium precipitation. Int. J. Dev. Neurosci. 18, 299-307.

Rodríguez, M.J., Martínez-Sanchez, M., Bernal, F., and Mahy, N. (2004). Heterogeneity between hippocampal and septal astroglia as a contributing factor to differential in vivo AMPA excitotoxicity. J. Neurosci. Res. 77, 344-353.

Rodríguez, M.J., Robledo, P., Andrade, C., and Mahy, N. (2005). In vivo co-ordinated interactions between inhibitory systems to control glutamate-mediated hippocampal excitability. J. Neurochem. 95, 651-661.

Rodríguez, M.J., Ursu, G., Bernal, F., Cusí, V., and Mahy, N. (2001). Perinatal human hypoxia-ischemia vulnerability correlates with brain calcification. Neurobiol. Dis. 8, 59-68.

Sapolsky, R.M. (2001). Cellular defenses against excitotoxic insults. J. Neurochem. 76, 1601-1611.

Saura, J., Boatell, M.L., Bendahan, G., and Mahy, N. (1995). Calcium deposits formation and glial reaction in rat brain after ibotenic acid-induced basal forebrain lesions. Eur. J. Neurosci. 7, 1569-1578.

Seifert, G., Schilling, K., and Steinhäuser, C. (2006). Astrocyte dysfunction in neurological disorders: a molecular perspective. Nat. Rev. Neurosci. 7, 194-206.

Sorrells, S.F., and Sapolsky, R.M. (2007). An inflammatory review of glucocorticoid actions in the CNS. Brain Behav. Immunity 21, 259-272.
Sousa, N., Lukoyanov, N.V., Madeira, M.D., Almeida, O.F., and Paula-Barbosa, M.M. (2000). Reorganization of the morphology of hippocampal neurites and synapses after stressinduced damage correlates with behavioral improvement. Neuroscience 97, 253-266.

Stefanova, N., Tison, F., Reindl, M., Poewe, W., and Wenning, G.K. (2005). Animal models of multiple system atrophy. Trends Neurosci. 28, 501-506.

Stein-Behrens, B.A., Elliott, E.M., Miller, C.A., Schilling, J.W., Newcombe, R., and Sapolsky, R.M. (1992). Glucocorticoids exacerbate kainic acid induced extracellular accumulation of excitatory amino acids in the rat hippocampus. J. Neurochem. $58,1730-1735$.

Stewart, D., and Vanderwolf, C.H. (1987). Hippocampal rhythmical slow activity following ibotenic acid lesions of the septal region. I. Relations to behaviour and effects of atropine and uretane. Brain Res. 423, 101-108.

Tata, D.A., Marciano, V.A., and Anderson, B.J. (2006). Synapse loss from chronically elevated glucocorticoids: Relationship to neuropil volume and cell number in hippocampal area CA3. J. Comp. Neurol. 498, 363-374.

Tomkins, O., Friedman, O., Ivens, S., Reiffurth, C., Major, S., Dreider, J.P., Heinemann, U., and Friedman, A. (2007). Bloodbrain barrier disruption results in delayed functional and structural alterations in the rat neocortex. Neurobiol. Dis. 25, 367-377.

van Landeghem, F.K.H., Weiss, T., Oehmichen, M., and von Deimling, A. (2006). Decreased expression of glutamate transporters in astrocytes after human traumatic brain injury. J. Neurotrauma 23, 1518-1528.

Waite, J., Chen, A., Wardlow, M., and Thal, L. (1994). Behavioural and biochemical consequences of combined lesions of the medial septum/diagonal band and nucleus basalis in the rat when ibotenic acid, quisqualic acid and AMPA are used. Exp. Neurol. 30, 214-229.

Yoshiyama, Y., Uryu, K., Higuchi, M., Longhi, L., Hoover, R., Fujimoto, S., McIntosh, T, Lee, V.M., and Trojanowski, J.Q. (2005). Enhanced neurofibrillary tangle formation, cerebral atrophy, and cognitive deficits induced by repetitive mild brain injury in transgenic tauopathy mouse model. J. Neurotrauma 22, 1134-1141.

Zapata, A., Capdevila, J., and Trullás, R. (2000). Role of highaffinity choline uptake on extracellular choline and acetylcholine evoked by NMDA. Synapse 35, 272-280.

Zlokovic, B.V. (2005). Neurovascular mechanisms of Alzheimer's neurodegeneration. Trends Neurosci. 28, 202-208.

Address correspondence to: Manuel J. Rodríguez, Ph.D. Unitat de Bioquímica

Facultat de Medicina, UB

c/Casanova 143

E-08036 Barcelona, Spain

E-mail: marodriguez@ub.edu 
This article has been cited by:

1. F.J. Ortega, J. Gimeno-Bayon, J.F. Espinosa-Parrilla, J.L. Carrasco, M. Batlle, M. Pugliese, N. Mahy, M.J. Rodríguez. 2012. ATP-dependent potassium channel blockade strengthens microglial neuroprotection after hypoxia-ischemia in rats. Experimental Neurology . [CrossRef] 